\title{
LA PRENSA COMO FUENTE PARA EL ESTUDIO DE LA HISTORIA DE LA EDUCACIÓN EN ESPAÑA DURANTE LA SEGUNDA MITAD DEL SIGLO XIX Y LA RESTAURACIÓN
}

\author{
The Press as a Source for the Study of the History of Education in Spain \\ During the Second Half of the 19th Century and the Restoration Period
}

\author{
Jorge Ramón Salinas \\ jramon@unizar.es \\ Universidad de Zaragoza. España \\ Fecha de recepción: 08/12/2019 \\ Fecha de aceptación: 03/03/2020
}

RESUMEN: En el presente artículo se reflexiona acerca del uso de la hemerografía como fuente para el estudio de la historia de la educación española, tanto en un aspecto general como en lo relacionado con las didácticas específicas (humanísticas, científicas, sociales, y artísticas) durante la segunda mitad del siglo XIX y los comienzos del siglo XX, abarcando también el periodo de la Restauración. En primer lugar, se expone el interés de este lapso temporal en el devenir histórico de la educación española y su relación con las publicaciones periódicas para, posteriormente, analizar su potencial informativo, discriminando entre las publicaciones periódicas generalistas y las especializadas. En última instancia se incide en la necesidad de abordar el uso de la hemerografía como fuente de investigación desde la interdisciplinariedad, siendo esencial una importante formación humanística y en Ciencias Sociales de aquellos investigadores que se sirvan de ella. Así mismo, tras exponer la situación actual de los repositorios digitales de prensa histórica y el aumento gradual de sus fondos, así como la facilidad en el acceso a las publicaciones periódicas propiciadas por las nuevas tecnologías, se plantean, para el periodo aludido, una serie de pautas procedimentales. Estas configuran una propuesta metodológica orientada a la adecuada utilización de la hemerografía histórica como fuente que nos permita desplegar todo su potencial informativo, aportando nuevos datos que propicien una mejor aproximación a la historia de la educación española.

Palabras clave: Historia de la Educación; prensa; siglo XIX; Restauración; didácticas específicas.

ABSTRACT: This article reflects on the use of hemerography as a source for the study of the history of education in Spain during the second half of the 19th century and the beginning of the 20th century, also covering the Restoration period, both from a global point of view and in relation to 
certain specific didactics (humanistic, scientific, social, and artistic). In the first place, the interest of this period is exposed in the historical evolution of Spanish education and its relationship with periodicals to subsequently analyse their informative possibilities, distinguishing generalist from specialized periodicals. In addition, this article emphasizes the need to address the use of hemerography as a source for research from interdisciplinarity, in its epistemological and methodological aspects as well as those related to the training of historians and / or pedagogues. Likewise, after exposing the current situation of the digital repositories of the historical press and the gradual increase of their collections, as well as the ease in accessing the periodical publications promoted by the new technologies, a series of procedural guidelines in relation to the aforementioned period are considered. These form a methodological proposal oriented to the adequate use of historical hemerography as a source that allows us to profit from its informative possibilities, thus contributing new data that propitiate a better approach to the history of Spanish education.

Keywords: History of spanish education; press; 19th century; Spanish Restoration period; specific didactics.

SUMARIO: 1. La historia de la educación española durante la segunda mitad del siglo xIX y la Restauración. La construcción del sistema educativo. 2. La hemerografía como fuente para la investigación: lenguaje, texto y discurso histórico. 3. La prensa española decimonónica y la Restauración: pautas metodológicas para su uso con fines de investigación. 3.1. La prensa pedagógica. 3.2. La prensa generalista y la historia de la educación durante la segunda mitad del siglo XIX y la Restauración. 4. Pautas metodológicos para el uso de la prensa como fuente en Historia de la Educación y de las didácticas específicas. 5 . El reto de la interdisciplinariedad en el uso de la prensa histórica como fuente en la Historia de la Educación. 6. Accesibilidad y redes de conocimiento: hemerotecas virtuales. 7. Conclusiones. 8. Referencias bibliográficas.

\section{LA HISTORIA DE LA EDUCACIÓN ESPAÑOLA DURANTE LA SEGUNDA MITAD DEL SIGLO XIX Y LA RESTAURACIÓN. LA CONSTRUCCIÓN DEL SISTEMA EDUCATIVO}

Uno de los periodos más relevantes en la historia de la educación contemporánea española se inicia en torno a la segunda mitad del siglo XIX y el primer cuarto del siglo xx, produciéndose hitos tan señalados como la aprobación de la Ley Moyano de 1857 o la creación del Ministerio de Instrucción Pública y Bellas Artes en 1900 (González y Muñoz, 2015, p. 263). Estos y otros hechos relevantes fueron gestados en un periodo caracterizado por profundos cambios socioeconómicos y constantes vaivenes políticos.

Tal y como apunta Juan Pablo Calero:

(...) el siglo XIX fue en España, en muy buena medida, el siglo de la educación. En pocas ocasiones, sobre todo si consideramos a la Segunda República como su epílogo, la educación ocupó un papel tan central en el debate político y entre las preocupaciones sociales. Dehechono creo que sea posible dejar de considerar al extraordinario renacimiento cultural que conocemos como «edad de plata» del arte, la ciencia y la literatura española sino como una forzosa consecuencia de la meritoria labor que desarrollaron maestros y profesores en las décadas anteriores. (Calero Delso, 2013, p. 315). 
El primer tercio del siglo xx constituye un periodo de evolución y afianzamiento de los planes educativos gestados en el siglo anterior, que se debatieron de forma polémica entre las nuevas tendencias renovadoras y el conservadurismo doctrinario, con el desarrollo de nuevos planteamientos teórico-pedagógicos y con una mejora en la gestión institucional y administrativa, materializada a través de la lenta introducción de mejoras en el plano laboral-corporativo del magisterio.

En el referido lapso temporal, y en especial durante todo el siglo XIX, se construyen los cimientos del sistema educativo moderno, impulsado por las ideas liberales de progreso. Estas estuvieron asentadas inicialmente en los clásicos principios ilustrados, tamizados por la idiosincrasia e intereses de las élites burguesas. Posteriormente la educación se renovaba desde los preceptos krausistas, y desde fines de siglo, por nuevos aires regeneracionistas. La legislación y el sistema educativo -a pesar de su espíritu laicista- habría de sobrellevar con altibajos y hasta la II República, la sempiterna vigilancia de la Iglesia, instaurada oficialmente desde el Concordato con la Santa Sede de 1851 (Cándido y Palacios, 1983, pp. 287-298).

La formación de la sociedad española se convertía en una cuestión de estado que se implementaba entre tiranteces conservadoras y liberales, las cuales no impidieron la redacción de diversas iniciativas legislativas como el Plan General de Estudios del ministro Pedro José Pidal de 1847 y, posteriormente, la redacción de la aludida Ley de Instrucción Pública de 1857 impulsada por Claudio Moyano (Miguel Alonso, 1990, p. 681-702 y Montero, 2009). Esta ley, de largo recorrido y vigencia, incluyó iniciativas anteriores, como las emprendidas en las cortes gaditanas en 1812, el Reglamento General de Instrucción Pública de 1821 de Antonio Remón Zarco del Valle y Huet, o el Plan General de Instrucción Pública firmado por Ángel de Saavedra, Duque de Rivas, en 1836 (Medina, 2012, p. 319).

La Ley Moyano, que respondía a los intereses de la nueva y pujante sociedad burguesa, era elitista en sus niveles secundario y superior, con aspectos muy conservadores, entre los que destacaba la supervisión moral eclesiástica. No obstante, desarrollaba una normativa legal que trajo consigo numerosos avances, tales como el establecimiento de la enseñanza centralizada, gratuita y obligatoria para niños y niñas, aunque con currículos segregados por géneros (Sarasúa, 2002, pp. 281-297). También establecía tres niveles educativos, la organización y normalización de la educación y sus agentes administrativos, ya fueran los ayuntamientos o el propio estado (Medina, 2012, p. 319). Del mismo modo, hacía imprescindible la carrera profesional de los docentes y por ende la difusión y mejora de las Escuelas Normales de Maestros y Maestras. Por desgracia, otro rasgo identitario de la segunda mitad del siglo XIX estaría en el incumplimiento de muchos de los compromisos marcados por la ley con estas instituciones, que llevarían a una grave precariedad económica y material del magisterio aún sufridos de forma endémica por los docentes hasta bien entrado el siglo $\mathrm{xx}$. 
Desde el último cuarto del siglo XIX o Primera Restauración (1875-1902) se producen posteriores concreciones y desarrollos del marco legislativo descrito iniciado en la Constitución de 1876 y otras iniciativas legales posteriores. Conforme avanzase el siglo y en los albores del siglo XX asistimos a la difusión de nuevas corrientes pedagógicas, especialmente las iniciativas de la Escuela Nueva y la Institución Libre de Enseñanza, como la creación del Museo de Instrucción Pública -luego Pedagógico Nacional-, la organización de las Conferencias Pedagógicas, la regulación de la enseñanza privada, la creación del Ministerio de Instrucción Pública y Bellas Artes, etc. que constituyen, entre otros, algunos de los temas destacados en el camino emprendido por la educación contemporánea la España. Todos ellos descritos por aportaciones historiográficas tan relevantes como los clásicos trabajos de Antonio Viñao Frago, Manuel Puelles Benítez, Olegario Negrín Fajardo o Alejandro Tiana Ferrer, entre otros.

Habida cuenta de todo lo expuesto, y aún a pesar del interés que también suscitan anteriores etapas de la historia de la educación, el periodo que abarca la segunda mitad del siglo XIX y la Restauración es un intervalo histórico de una densa y trascendente actividad en el ámbito educativo español. Este desarrollo instructivo-pedagógico discurre paralelo al de la expansión y propagación de la prensa española. Las publicaciones periódicas se convierten, al igual que los procesos de industrialización, en elementos distintivos de la propia sociedad decimonónica e intersecular. Esta profusión de publicaciones, en forma de prensa generalista y prensa especializada, continuaría desarrollándose de forma intensa conforme avanzase el siglo xx, incrementando su alcance y distribución, a la vez que su espectro de consumidores. Dentro de esta "eclosión hemerográfica», la educación y sus protagonistas, convertidos en temas de actualidad e interés social de un gran calado político, iban a generar «auténticos ríos de tinta» en forma de publicaciones especializadas diversas, así como en referencias recurrentes en los periódicos de información general.

De este modo y como veremos, este medio de comunicación de masas constituye una valiosa y sólida fuente de información para el investigador, sobre cuyo potencial como fuente para la investigación -plenamente asumido en la actualidad-disertaremos a continuación. En primer lugar, analizaremos la naturaleza de la información contenida en la hemerografía, y posteriormente plantearemos diversas pautas procedimentales y metodológicas para su utilización como fuente de estudio durante el periodo aludido.

\section{LA HEMEROGRAFÍA COMO FUENTE PARA LA INVESTIGACIÓN: LENGUAJE, TEXTO Y DISCURSO HISTÓRICO}

Queda asumido desde el punto de vista epistemológico el carácter científico de la Historia, y su construcción a partir de sus fuentes, diversas y categorizadas tradicionalmente $-y$ todavía con vigencia- según J. G. Droysen (1808-1884) en 
«monumentos», «restos» y "otras fuentes escritas o textuales» (Lozano, 2015, p. 84 y Navarro, 2002, pp. 39-67).

No obstante, cabe señalar la existencia de diversos debates en torno al papel del lenguaje en el discurso histórico en los que se diserta sobre la propia naturaleza y limitaciones del mismo (Viñao Frago, 1996, p. 159 y Lozano, 2015). La reflexión sobre su propia configuración ha suscitado numerosas inquietudes surgidas cuando se pretende, a través de la interpretación de textos históricos, recobrar realmente eventos y acciones pasadas.

Para algunos investigadores la validez del lenguaje utilizado en las fuentes textuales debe ser matizada. Son numerosas y profusas las interpretaciones sobre las particularidades y limitaciones del propio lenguaje escrito como vehículo de transmisión y comunicación en el discurso histórico aunque, en general, se lo considere como un reflejo o «huella de un referente de la realidad que se inscribe en el lenguaje y en su configuración material» (Viñao Frago, 1996, p. 165).

Al margen de las referidas matizaciones en torno a la naturaleza del propio texto histórico, la hemerografía se ha convertido en objeto de estudio por parte de numerosos investigadores de diferentes disciplinas, especialmente aquellos vinculados con la humanidades y las ciencias sociales. Su uso como fuente documental trae consigo la apertura de diferentes vías de conocimiento: por una parte, la utilización de la prensa como fuente genérica de documentación, complementaria al uso de otras sobre determinados hechos, así como sobre la opinión pública suscitada en torno a ellos. Por otra, el uso de la hemerografía como fuente de documentación sobre grupos y categorías sociales. Especialmente relevante en el caso de las publicaciones especializadas, y sobre todo, en el de la prensa profesional (Duverger, 1996, pp. 122-123).

La historiografía contemporánea ha asumido el potencial informativo de las publicaciones periódicas para el estudio de los procesos históricos, destacando su papel para abordar la comprensión del pasado (Dolores Saíz, 1996, p. 132). Una tarea en la que los investigadores llevan a cabo una aproximación a los hechos pretéritos desde vestigios sesgados de diversa naturaleza, testimonios fragmentarios que el historiador pretende recomponer y actualizar ayudado de múltiples herramientas metodológicas y procedimientos propios de las ciencias (Lozano, 2015, p. 77).

La prensa adquiere una doble dimensión en el proceso de los estudios del pasado, al constitutir un «objeto histórico» en sí misma, a la vez que presenta una información esencial como testimonio de un momento concreto (Ramón Salinas, 2014, p. 15). Se trata de «instantáneas textuales» de diversa naturaleza creadas como literatura de consumo, un tanto efímera -sobre todo en el caso de la prensa diaria en la que puede desprenderse una gran cantidad de información que el historiador debe analizar, clasificar e interpretar.

Por otra parte, debemos destacar que en la hemerografía se hace patente la importancia de la dimensión contextual del discurso histórico, más allá de las 
particularidades y tipologías del mismo. De hecho, todos los textos nos remiten ineludiblemente a diversos contextos (Viñao Frago, 1996, pp. 171 y 169):

(...) Al del autor, por supuesto, pero también a los de quienes de forma material o inmaterial incorporan sentidos al mismo y a los de quienes se apropian de él. Y estos contextos no son sólo textuales, sino también sociales, materiales y, en lo que al lenguaje se refiere, de índole oral, entre otros modos de representación de lo real. (...) Todo texto remite a varios contextos. A los de producción e incorporación de sentidos, y a los de recepción e interpretación. Los primeros se refieren no sólo al autor, sino también a todos aquellos -impresores, editores, libreros, lectores anteriores, comentaristas- que con su actividad hacen posible o dificultan una u otra apropiación, uno u otro sentido.

En realidad, la propia definición de la Historia trae implícita la interpretación del contexto histórico del discurso y su estudio crítico, que ha sido desarrollado especialmente por el profesor Teun A. Van Dijk (Van Dijk, 2017, p. 33; 2011; 2003; et alia). En consecuencia, la prensa se convierte en depositaria de ese discurso cuyo análisis debería ser abordado de forma exhaustiva.

La labor del historiador se convierte en una recomposición de diferentes fuentes de información fragmentarias y dispersas en las que la hemerografía puede alcanzar gran relevancia. Las publicaciones periódicas son elementos insertos en el marco coyuntural en el que se producen y son reflejo de los avatares de un momento socio-histórico concreto.

En los ya clásicos trabajos sobre la prensa como fuente histórica de Manuel Tuñón de Lara, se subraya que la hemerografía constituye una herramienta polivalente de primer orden: puede ser una fuente de información sobre cuestiones precisas, o una fuente para expresar corrientes de opinión, actitudes políticas o ideológicas. También una fuente que recoge las mentalidades de una época, desde la citada información política hasta la económica, desde la difusión cultural al ensayo literario, incluyendo los gustos y las modas hasta los más serios debates (Tuñón de Lara, 1974 pp. 21 y 174). De este modo podemos afirmar que cuando se hace historia de la prensa se está haciendo a la vez historia de las fuentes (Salinas, 1995, pp. 280-281) ya que la propia prensa se convierte en una fuente de información determinante sobre ella misma.

La naturaleza de la hemerografía histórica adquiere mayor interés puesto que el investigador ha de completar el análisis de la información obtenida con el estudio de esta como objeto histórico (Duverger, 1996, p. 124). El manejo de la misma implica conocer el contexto de cada publicación, así como sus particularidades, es decir, comprender la trama coyuntural que la propia prensa retrata y de la que simultáneamente se convierte en un elemento más en la configuración de la misma. Esto es así hasta el punto de que el estudio sistemático de la historia de la hemerografía, tanto en aspectos formales y estructurales, así como en 
su contenido, posibilita la comprensión de la historia contemporánea en general (Desvois,1986, p. 359).

Por el contrario, una utilización superficial de la prensa como fuente puede conducir en ocasiones a una distorsión de la información obtenida:

Estudiar el contenido, sin conocer previamente todos los elementos anteriores, es como es como levantar un castillo sobre arenas movedizas de donde se deduce que la prensa antes de ser utilizada como fuente histórica, requiere un complejo estudio previo para colocar al portavoz en el centro de sus propias coordenadas. Sólo así el rico contenido que ofrecen los medios de comunicación de masas adquiere pleno sentido y se convierte en una fuente histórica de capital importancia. En otro caso, cuando menos, estamos ante una parcialidad con pretensiones de visión global. (Alumiña, 1997, p. 110).

Un mal uso de las fuentes históricas podría hacernos incurrir en los típicos errores citados por Jorge Lozano, y que según apunta el investigador ya habrían sido a su vez observados en el siglo xIV por el historiador y teórico, Abderrahman Ibn Jaldún (1332-1406). Estos se producen cuando se realiza una inadecuada aproximación a los hechos históricos (Lozano, 2015, pp. 82-83 y Vivanco, 2000, pp. 27-43):

- La parcialidad hacia un ideario, el exceso de confianza en la fiabilidad de las fuentes, la falta de comprensión respecto a la significación de un hecho.

- La falta de habilidad para encuadrar un acontecimiento dentro de su contexto real.

- La ignorancia de las leyes que gobiernan la transformación de la sociedad humana.

- El afán de tergiversar la interpretación histórica por el deseo, de ganarse el favor de cargos e instituciones.

Así pues, en aras de alcanzar el presupuesto deseo de independencia e imparcialidad deontológica del historiador -rasgo que necesariamente debiera ser consustancial al mismo- resulta oportuno, tal y como recuerda Enrique Moradiellos, recordar las palabras de Cornelio Tácito (55-ca.117): «la historia sólo se escribe bona fides, sine ira et studio, es decir, "con buena fé interpretativa de partida, sin encono partidista sectario y tras meditada reflexión sobre los materiales probatorios disponibles» (Moradiellos, 2016, p. 29). Del mismo modo y para realizar un adecuado trabajo de investigación, se hace necesaria una cauta aproximación a la fuente, procediendo a la realización de un proceso de análisis pormenorizado de la publicación en aras de interpretar correctamente su contenido (Hernández Ramos, 2017, p. 476). El contraste con otras fuentes se convierte en una necesidad consustancial al uso de la prensa, ya que esta se presenta con relativa frecuencia cargada de subjetividad, ideologías e intencionalidades ulteriores que distorsionan frecuentemente el objeto de estudio (Arroyo, 2004, pp. 435-446 y Arostegui, 2004, pp. 68-71). 


\section{LA PRENSA ESPAÑOLA DECIMONÓNICA Y LA RESTAURACIÓN: PAUTAS METODOLÓGICAS PARA SU USO CON FINES DE INVESTIGACIÓN}

La prensa española se convertía, tras sus fases iniciales o preperiodísticas y su evolución en los siglos XVII y XVIII en el exponente de una nueva cultura urbana burguesa. Inicialmente fue una herramienta de propaganda regia barroca primero, e ilustrada después (Espejo, 2013, pp. 11-14). Existe un punto claro de inflexión a partir de 1808 (Seoane, 1996, p. 19), en el que comenzaba una nueva etapa de desarrollo durante la primera mitad del siglo $x I X$, adquiriendo paulatinamente una creciente dimensión política y empresarial. Con posterioridad aumentaría su pujanza en la segunda mitad del ochocientos, y especialmente durante su último cuarto, con el que daba inicio la Restauración, y con ella, una coyuntura favorable para un mayor auge hemerográfico.

En la prensa de este último periodo, marcado por la reposición de la monarquía borbónica, abundaron, paralelamente a este proceso de difusión y expansión, los temas referidos a la educación y su casuística, convertidos en asuntos de actualidad socialmente relevantes, abordados desde todas las tendencias político-ideológicas. Sirvan de ejemplo en este sentido las aportaciones de José M. a Nasarre y Pilar Teruel sobre cuestiones educativas en la hemerografía oscense concretamente las obtenidas en El Diario de Huesca entre 1875 y 1902 (Nasarre, 2001, p. 143; Teruel, 2001, p. 203).

En torno al sexenio bélico napoleónico (1808-1814) alrededor del $40 \%$ de los títulos de prensa españoles se imprimían en Andalucía (Checa Godoy, 2011, p. 32). El proceso de desarrollo de la prensa se produciría en las principales poblaciones urbanas españolas, aunque Madrid se asentaría fundamentalmente como capital del periodismo español decimonónico. Conforme avanzase el siglo XIX se prodigaron progresivamente en España gran multitud de títulos hemerográficos espoleados por el desarrollo urbano y la pujanza demográfica, económica y política que se incrementarían sustancialmente desde la segunda mitad del mismo. En la década de 1860 el negocio de la prensa se basaba fundamentalmente en la venta directa en la propia calle, mientras que en la de 1880 quedaría patente la estabilidad económica necesaria para establecer una presión mediática independiente (Mediavilla, 2009, p. 82). La difusión a través del correo y el ferrocarril habrían permitido la divulgación hemerográfica por todo el territorio peninsular, vertebrado por la demarcación liberal en provincias desde 1833. Las publicaciones periódicas del siglo XIX mostrarían paulatinamente una incipiente madurez, con una tirada significativa y en crecimiento, un precio asequible y un lenguaje renovado. La prensa generalista se habría afianzado entre la dirección y el control de determinados intereses políticos de variado signo y la intención de fomentar la rentabilidad del negocio editorial con desiguales resultados (Mediavilla, 2009, p. 82). 


\subsection{La prensa pedagógica}

El desarrollo de la prensa y el interés creciente en la esfera pública por la educación iba a favorecer el desarrollo de una especialización monográfica. Así, desde la segunda mitad del siglo XIX, iniciada una etapa destacada en la construcción del sistema educativo español, emprendían gradualmente su andadura diversas publicaciones periódicas profesionales dedicadas a la educación con diferentes periodicidades. Suscribiendo las palabras de José M. a Hernández, esta prensa puede definirse como pedagógica:

(...) por el objeto que la califica, va a ir construyendo sus señas de identidad en función de la complejidad que representan los procesos de construcción y vida activa del sistema escolar y de otras instituciones e iniciativas educativas propias de la comunidad y sus instituciones, públicas y particulares (...). El carácter patrimonial de la prensa pedagógica, y su inserción en el concepto de patrimonio histórico educativo, se inscribe en el enriquecimiento cuantitativo y cualitativo del concepto de fuente para la investigación histórico educativa. Si hace alguna generación se discutía y cuestionaba el valor y fidelidad de otras fuentes que no fueran documentos inéditos de archivo relativos a la educación, hace ya algunos decenios que fueron admitiéndose casi de forma canónica otras fuentes documentales, convenientemente depuradas, de procedencia icónica, oral, material, y desde luego la prensa y publicaciones periódicas relativas a temática educativa y pedagógica. (Hernández, 2013, pp. 12-15).

Los estudios de historia de la educación sobre la prensa pedagógica o especializada se han incrementado recientemente. Muchos de ellos se centran en trabajos de ámbito local de diversa entidad -Teruel, Badajoz, Valladolid, Canarias, Asturias, Cantabria, etcétera- (Hernández, 2013, pp. 15-32).

La prensa pedagógica a nivel nacional también constituye una fuente fundamental para el historiador de la educación, quien debería abordarla en primera instancia para, posteriormente, "descender» a la circunscrita a un ámbito territorial reducido. En este sentido, la hemerografía educativa local resulta igualmente interesante y permite conocer el nivel de concreción de diversos aspectos relacionados con el marco educativo general.

Hasta el último cuarto del siglo XIX no comenzaron a generarse en España un número significativo de periódicos referentes al magisterio. Este número aumentaría conforme el sistema político de la Restauración se fuera consolidando. Este periodo favoreció la estabilidad, así como un renovado clima de tolerancia, que supuso "una primera etapa dorada para la prensa educativa hispana», en la que convivieron, no sin polémica y dentro de la normalización legal que supuso la Ley Moyano de 1857, diversas posiciones político-ideológicas, laicistas y católicas, estas últimas favorecidas desde el concordato con la Santa Sede de 1851 (Checa, 2002, pp. 22-23). 
Estas tendencias establecieron en el ámbito de la educación -siendo conscientes de su poder determinante en la construcción ideológica de la sociedad- un nuevo escenario de enfrentamiento, arrastrado desde principios del siglo XIX. Los conflictos entre republicanos de diversas tendencias y los sectores monárquicos; entre la burguesía, las clases medias y los sectores sociales más humildes; entre liberales y conservadores, laicistas y católicos de diverso calado, así como otras divisiones, se tradujeron de forma constante en el ámbito educativo (Ruíz y Palacios, 1983, p. 289).

No obstante, en este periodo convulso y polémico de la educación española también se alcanzarían diversos logros, promovidos por el intenso debate sobre estas cuestiones. De este modo encontramos iniciativas que marcarían el posterior desarrollo pedagógico de la nación, como la creación de la citada Institución Libre de Enseñanza, las Escuelas del Ave María de Andrés Manjón (Moreno, 2010) los Congresos Pedagógicos de las décadas de 1880 y 1890 del siglo xIX (Ruíz, 1980, pp. 401-422), así como la aparición del Museo Pedagógico Nacional, entre otros hitos. En los Congresos Pedagógicos nacionales, con un interés más teórico y científico, no faltarían las demandas ante la grave crisis que atravesaría el sector a merced de la cambiante deriva política (Checa, 2002, p. 24).

Así pues, entre 1875 y los primeros años del siglo xx -lo que la historiografía denomina tradicionalmente como la Primera Restauración (1875-1902) (Jover, 1993, pp. 271-73; Jover, Gómez y Fusi, 2003)- vemos aumentado el número de publicaciones pedagógicas traspasando los cincuenta títulos en la década de 1880 y los setenta en la siguiente. Se trataría concretamente, según Checa Godoy (2002, pp. 22-23) de diversas publicaciones que podemos agrupar grosso modo en:

- Revistas profesionales del magisterio, la mayoría de talante laico y liberal, y muchas de carácter provincial. Sin embargo, también abundaron un reseñable número de estas pertenecientes a los sectores católicos, cuyo papel en el panorama educativo de la Restauración fue muy relevante:

(...) irán surgiendo, espoleadas por las circunstancias políticas, iniciativas de carácter confesional y conservador. Se trata de publicaciones que en buena medida, se gestan como respuesta y contrapeso al sesgo liberal y laico que predomina en el mercado de la prensa educativa. Se va fraguando de este modo, un espacio de opinión contrapuesto al discurso educativo liberal. (Mediavilla, 2009, p. 95).

La nueva deriva ideológica de la Iglesia se abría tras el Concilio Vaticano I, y desde la revolución de 1868, propiciaba en España la aparición de numerosos periódicos, revistas y folletos católicos. Inicialmente la Iglesia arremetía sistemáticamente contra la prensa -durante la primera mitad del siglo XIX-como divulgadora de preceptos liberales y secularizadores. Posteriormente la Iglesia cambiaba de estrategia, y la prensa católica se convertía, ya en la segunda mitad del siglo, en una 
nueva y poderosa herramienta difusora de su ideario. (Hibbs-Lissorgués, 1995, pp. 71 y ss.) En estas publicaciones católico-pedagógicas se priorizaba la transmisión de su pensamiento por encima de la defensa de intereses laborales y profesionales, así como de reflexiones acerca de la renovación del sector.

- Publicaciones de colegios privados no religiosos, que continúan la impronta del influyente boletín de la I. L. E. madrileña y sus modelos educativos liberales que tendría destacada difusión e influencia.

- Publicaciones realizadas por el alumnado de centros públicos y privados, estudiantes, colegiales y escolares (Hernández Díaz, 2015, p. 13; y Checa, 2002).

El referido Antonio Checa, referencia imprescindible en el tema que nos ocupa, señala que a finales de siglo XIX son muchas las ciudades españolas en las que se dictan dos periódicos pedagógicos, de significación católica uno, y liberal o sencillamente laico otro (Checa, 2002, pp. 22-29). Del mismo modo apunta que hasta comienzos del siglo xx lo más abundante sigue siendo la prensa fomentada por maestros y dirigida a maestros. Estas publicaciones, que en algunos casos serían sorprendentemente duraderas, tendrían una configuración muy parecida, y se dedicaron fundamentalmente a describir los problemas del ramo. A estos se dedica la mayor parte de sus páginas, cargadas de mensajes pesimistas, y de constantes quejas a la administración, fundamentalmente la local, como principal responsable de las carencias materiales y económicas sufridas con especial énfasis hasta los primeros años del siglo XX, momento en el que el estado asumió el pago de los salarios, dependientes hasta ese momento de los Ayuntamientos. Con esta medida, los docentes consiguieron solventar en gran medida los constantes impagos que padecían, sumiéndolos frecuentemente en la miseria. La administración estatal quedaría también encargada de la acreditación de los profesionales del sector.

Todas estas revistas y periódicos son consideradas en su inmensa mayoría como representantes declarados del profesorado provincial de primera enseñanza. Son escasas las manifiestamente católicas, aunque el anticlericalismo está prácticamente ausente de las mismas (Checa, 2002, 22-23).

Una vez entrado el siglo $x x$, la prensa educativa comenzaba a enriquecerse con las aportaciones teóricas de sectores independientes, innovadores y entonces vanguardistas, alejados de las corrientes educativas imperantes, como las reflexiones de Juan Benejam o los modelos libertarios de las ideas de Ferrer y Guardia. La renovación desde posiciones vinculadas al catolicismo vendría fundamentalmente de la mano del Padre Andrés Manjón y Manjón. Todos ellos cristalizaron sus ideas en publicaciones y en prensa de diversa naturaleza (Checa, 2002, 27).

Con la llegada del siglo xx y la mejora lenta pero progresiva de la situación económica de los docentes, la prensa educativa diversificaría paulatinamente sus contenidos dedicándose también a temas pedagógicos y no solo laborales. Este avance en el poder adquisitivo del sector supuso un gran estímulo para el mercado editorial de la prensa educativa (Mediavilla, 2009, p. 93). 
En resumen, podemos afirmar que el paréntesis temporal intersecular que abarca el último cuarto del siglo xIX y principios del Xx supuso un periodo en el que se prodigaron las publicaciones periódicas que abordaron la educación desde un punto de vista también teórico, aunque preponderantemente corporativo y laboral. Sería en esta etapa en la que el sector alcanzaría sus principales logros profesionales. La prensa dedicada al magisterio constituye la mayor parte del corpus periodístico educativo, creado desde diversos agentes, instituciones e ideologías.

La abundancia de títulos de prensa educativa en estos años, algunos de ellos de vida efímera, indica que se trata en ocasiones de publicaciones de escaso recorrido debido a las penurias económicas del colectivo. No obstante, su profusión y la contrastante longevidad de otras muchas -como El Magisterio Español, o La Enseñanza Católica-, muestra por otra parte una insistencia y un afán reivindicativo ante la crítica situación del sector (Hernández, 1983, p. 68).

Conforme avanzase el siglo xx las publicaciones periódicas pedagógicas ya no iban a tratar en exclusiva sobre las demandas habituales de los docentes ante las distintas administraciones. Fueron abordadas otras cuestiones, como la formación de los profesionales, generándose una prensa mucho más centrada en la pedagogía que en el ámbito profesional corporativo. De forma paulatina y al mismo tiempo se irían polarizando dos tendencias en el ámbito educativo: la conservadora y la renovadora. Asimismo, las primeras décadas del siglo Xx constituyen por una parte un gran avance de la enseñanza religiosa en España y, por otra, un aumento de la referida politización de la enseñanza, trasladándose a la escuela la división política e ideológica de la sociedad (Mediavilla, 2009, p. 97 y Checa, 2002, p. 29).

En la mayoría de las ocasiones fueron los mismos docentes los que, imbuidos de regeneración y cambio unos, y de continuismo doctrinario otros, crearon y dirigieron sus propias publicaciones profesionales desde diversa perspectiva. En otras ocasiones fueron las propias instituciones educativas, públicas o privadas -sirva de ejemplo el citado Boletín de la Institución Libre de Enseñanza- las que propiciaron la impresión de su propia prensa dando a conocer sus trabajos, y demandando mejoras, así como cambios en una profesión, en muchas ocasiones, de difícil desempeño.

La prensa especializada, cuya profusión se incrementaría, ampliando y variando sus contenidos durante el primer tercio del siglo xx (Borroy, 2004, p. 32) aporta por razones obvias- una gran cantidad de datos acerca de la educación y la práctica. Su papel como fuente de información es muy relevante para el estudio de la historia de la educación y también de las didácticas específicas (Ezpeleta, 2015-2016, pp. 159-171).

No obstante, y como veremos seguidamente, no debe infravalorarse todo el elenco de referencias directas e indirectas que puede proporcionarnos el estudio de la prensa generalista. Por ello deben establecerse una serie de pautas de 
investigación que nos permitan extraer todo su potencial informativo y abordar adecuadamente los contenidos fundamentales relacionados con la historia de la educación. Las características de la hemerografía y su conexión con el devenir sociopolítico y cultural del periodo estudiado hacen necesaria una reflexión acerca de las necesidades formativas del investigador que desea interpretar la información allí contenida y sobre la que nos referiremos a continuación.

\subsection{La prensa generalista y la historia de la educación durante la segunda mitad del siglo xIX y la Restauración}

Todos estos aspectos que acabamos de reseñar para la prensa educativa especializada se reflejaron también en la prensa generalista española, que registraba entre sus páginas las diversas opiniones y tendencias sobre el ámbito educativo, convertido como sabemos en un escenario recurrente de enfrentamiento político. Más aún teniendo en cuenta el cariz profundamente tendencioso de la mayor parte de la prensa periódica, marcada por las diversas posiciones ideológicas imperantes. Permanecería en ella, no obstante, un espíritu de divulgación cultural y de captación de un espectro de lectores cada vez más amplio, favorecido por el cese de la censura propiciado por la legislación liberal del primer gobierno sagastino -Ley Gullón de 1883 (Marcuello, 1999, p. 86)-.

A comienzos del siglo xx se mantenía en la sociedad española -todavía estratificada y sufriendo de forma desigual el progreso de la enseñanza- la consideración generalizada de la prensa (...) «como lugar de una expresión significadora y de apoyo a la confirmación de nuevas vanguardias intelectuales y políticas» (Bussy Genevois, 2002, pp. 383-384).

Los temas que sobre educación se reflejaron en la prensa generalista fueron diversos, muchos de ellos comunes a los abordados en la prensa profesional especializada, y podrían agruparse en las siguientes categorías:

- En primer lugar, destacan aquellos centrados en recurrentes reivindicaciones laborales y denuncias sobre las penurias y carencias materiales y humanas en el ámbito educativo, sobre todo de la escuela primaria.

- Otro conjunto determinante de datos sería de tipo administrativo- informativo: oposiciones y concursos, traslados de personal, tomas de posesión, nombramientos y ceses, cierre o apertura de escuelas, premios obtenidos en exámenes públicos, actos diversos de inicio o final de curso, etc.

- Por otra parte, encontramos un interesante conjunto de referencias indirectas, a las que luego nos referiremos y que se obtienen tras una detallada revisión de los ejemplares conservados.

El reconocimiento que alcanzaba el magisterio a lo largo de las primeras décadas del siglo xx estaría ligado a la mayor presencia de los maestros en la sociedad, 
fundamentalmente a través de tres aspectos, entre ellos su papel activo en el mundo periodístico:

En primer lugar, de la prensa local que fue un escaparate para proyectar sus inquietudes, sus intereses y sus conocimientos. Los maestros firmaron artículos sobre los temas más variados, desde cuestiones de actualidad, hasta artículos históricos, de costumbres, etc. En otras ocasiones, fueron las conferencias (pedagógicas) el pretexto para proyectarse en la sociedad. En tercer lugar, los maestros publicaron, durante este período, un buen número de libros para las escuelas: gramáticas, aritméticas, libros de lectura, etc., pero, además, durante el primer tercio del siglo $x x$ algunos maestros escribieron obras dirigidas al gran público. (Borroy, 2004, p. 32).

Estas producciones literarias se convierten en valiosos conjuntos documentales. Su análisis constituye una importante fuente de datos para profundizar en el ámbito de la historia de la educación, incluidos determinados aspectos referidos a procedimientos didácticos utilizados en las diversas áreas de conocimiento. La prensa recoge, en efecto, información variada que directa o indirectamente nos conduce a establecer conjeturas más o menos plausibles acerca de la praxis educativa de los docentes. En este sentido la importancia de la prensa histórica generalista como fuente podría explicarse atendiendo fundamentalmente a los siguientes aspectos:

- Muchos maestros y profesores dirigieron y colaboraron asiduamente en publicaciones locales: periódicos y revistas, de diverso contenido en temas educativos, y de variado signo político. En ellas, volcarían frecuentemente sus opiniones, ideario y tendencias pedagógico-profesionales.

- La publicidad de centros educativos, especialmente los privados, cuya importancia ha sido reseñada entre otros por Alejandro Tiana (Tiana Ferrer, 1994, p. 119), aparece publicitada en los diarios, y nos permite el conocimiento de sus protagonistas: equipo docente, espacios-locales, condiciones de los mismos, especialidades, asignaturas, cuestiones curriculares, tarifas, actividades complementarias, calendario escolar, etc.

- Referencias a la utilización de manuales y libros de texto, obra de los propios docentes o de otros autores, que, una vez localizados y analizados, nos remiten a conocer diversos procedimientos sobre didácticas específicas.

Para propiciar una correcta utilización de la prensa generalista como fuente con fines de investigación resulta esencial la revisión minuciosa de los ejemplares conservados, pudiendo ser obtenidos contenidos como los siguientes, con independencia del género periodístico en que se encuentren y la sección del periódico estudiado: 
- Opiniones, tendencias ideológicas y educación. Modelos, referentes y contenidos.

- Referencias a encuentros, reuniones y sociedades culturales y/o pedagógicas.

- Alusiones a aspectos legislativos, problemas reales y carencias en su ámbito de aplicación. Denuncias por incumplimiento del marcolegal establecido: libramientos, provisión de plazas, concursos, etc., así como de otras irregularidades.

- Necesidades humanas y materiales: número de colegios, exceso de alumnos, ratios, etc.

- Agentes promotores, personal docente y laboral de colegios públicos y privados de primera y segunda enseñanza, así como de otros estudios especiales.

- Espacios, estatutos, ideario y directrices de los directores. Resultados académicos, horarios, actividades, servicios, etc.

- Relaciones entre el magisterio y la docencia, el periodismo y la esfera social, política y cultural locales.

\section{PAUTAS METOdOLÓGICOS PARA EL USO DE LA PRENSA COMO FUENTE EN HISTORIA DE LA EDUCACIÓN Y DE LAS DIDÁCTICAS ESPECÍFICAS}

A la hora de abordar el estudio concreto de la historia de la educación en un periodo histórico concreto como el que nos ocupa a través de la prensa es recomendable seguir las directrices específicas de la investigación histórica, es decir, un planteamiento historiográfico basado en el adecuado uso de diversos instrumentos de análisis histórico. En este, debieran tenerse en cuenta los diferentes elementos primordiales para alcanzar el objetivo de una investigación histórica: la exposición de una secuencia temporal y de un espacio socio-histórico claramente definidos, un análisis social global, y el estudio de los fenómenos socio-históricos particulares.

Así pues, debemos tener en cuenta «diversos planos o perspectivas de cuyo entrecruzamiento y conexión derivaremos en una serie de ideas plausibles distintas, de investigaciones posibles y de complejidad metodológica, en suma, de la historiografía» (Arostegui, 2001, pp. 320-321).

Los datos obtenidos, no tan solo aquellos específicamente referidos del ámbito educativo y de la historia de la educación, nos conducen a una necesaria perspectiva sistemática multidisciplinar, que versa sobre diversos temas individualizados entendidos y relacionados dentro del marco contextual.

Dentro de este proceso, el investigador debe realizar una revisión detallada de los ejemplares seleccionados, incluyendo la prensa generalista, puesto que en esta la información puede localizarse en cualquier parte, desde la cabecera hasta la sección de los anuncios, habitualmente insertos en las últimas páginas.

Debe tenerse en cuenta el tiempo y esfuerzo necesarios en la revisión minuciosa de la hemerografía conservada, dada la diversidad de una información que, como decimos, puede aparecer en cualquier sección de las publicaciones y que 
en muchas ocasiones permanece todavía «invisible» a las modernas herramientas de búsqueda textual. Una "batida» rigurosa y sistemática sobre las fuentes hemerográficas permite el hallazgo de datos referidos a personajes, espacios, materiales, etc., que complementan lo obtenido en los textos más accesibles. Es esta información dispersa, en apariencia tangencial y ocasionalmente casi accidental, la que pasa desapercibida al investigador acostumbrado a la prensa especializada y a un tratamiento inmediato y monográfico de los contenidos. Sirvan de ejemplo las aficiones velocipédicas de un maestro en una lista de participantes en un evento deportivo, su pertenencia a una sociedad de ocio, su aparición en colectas y suscripciones públicas, o un minúsculo anuncio de la venta de mobiliario de un colegio privado por cierre del mismo.

Toda esta información puede ser relevante y, en muchas ocasiones, permite establecer relaciones diversas entre aspectos propios del sector educativo y los factores contextuales. Los datos recogidos hacen posible conformar, de forma interdisciplinar, una realidad pedagógica diversa y compleja, intrínsecamente relacionada con el resto de los ámbitos de la sociedad que la genera, facilitando su comprensión.

\section{EL RETO DE LA INTERDISCIPLINARIEDAD EN EL USO DE LA PRENSA HISTÓRICA COMO FUENTE EN LA HISTORIA DE LA EDUCACIÓN}

Tal y como acabamos de relatar, la interdisciplinariedad se convierte en una dinámica necesaria dentro del proceso metodológico descrito. La naturaleza diversa de la propia información contenida en la prensa así lo requiere.

En palabras del historiador oscense Julio Brioso:

(...) cuantos investigamos en prensa conocemos lo fatigoso que resulta consultarla y el tiempo que en ello se invierte, cuando van surgiendo, al propio tiempo, multitud de temas interesantes que van distrayendo y apartando del principal objeto de estudio. (Brioso, 2001, pp. 55-84).

No obstante, esta circunstancia apuntada por Brioso refleja la «riqueza» informativa de la propia fuente, y que a su vez permite al investigador establecer relaciones en varias direcciones creando así las referidas redes de conocimiento multidisciplinar e interdisciplinar. Por ello, se hace necesaria una sólida formación histórica y humanística del investigador, ya que los factores coyunturales son determinantes en la pretensión de realizar una aproximación certera al objeto de nuestro estudio. Destacaremos a grandes rasgos los siguientes: 
- Conocimiento de la situación socio-política, cultural y económica del periodo estudiado. Tendencia político ideológica de las publicaciones. Impresores, aspectos formales, directores, redactores habituales, colaboradores y línea editorial.

- Lugar de publicación, características urbanas y de la población propias del ámbito geográfico y humano.

- Elementos definitorios de cada publicación: historia, propietarios, gerentes, orientadores ideológicos, distribución e impacto, clientelas, contenidos, etc.

- A todos estos han de sumarse los aspectos formales: páginas, maquetación, secciones, ilustraciones (si las hubiese), etc. relacionándolos con todo lo anteriormente señalado.

Estos elementos derivados del carácter contextual del discurso histórico redundan nuevamente en el perfil del investigador que ha de ser capaz de relacionar cuestiones, no solamente propias de su área de conocimiento específica, sino que debe poder conectar de forma conjunta toda una imbricada red de relaciones de conceptos y procesos sociológicos, históricos y humanísticos. Solo así la información impresa presenta y genera todo su potencial como fuente de conocimiento.

\section{ACCESIBILIDAD Y REDES DE CONOCIMIENTO: HEMEROTECAS VIRTUALES}

La mejor abordabilidad de las fuentes hemerográficas ha favorecido la aparición de trabajos basados en el uso de este recurso como fuente histórica. La conservación de la prensa es uno de los principales retos de la biblioteconomía, en la que los avances propiciados por el uso de las nuevas tecnologías permite su progresiva divulgación y catalogación. Además de la digitalización, perviven con paradójica vigencia en algunas instituciones -tales como bibliotecas nacionales y los grandes consorcios internacionales- algunos métodos tradicionales como la microfilmación, debido, entre otras ventajas, a su contrastada durabilidad (Abadal y Guallar, 2010, p. 141).

Las diversas colecciones de publicaciones periódicas generalistas y especializadas albergadas en diferentes plataformas de divulgación bibliográfica han facilitado el acceso a este recurso. Las bibliotecas y otras instituciones, públicas y privadas, han puesto a disposición del investigador un número creciente de prensa histórica a través de los citados procesos de digitalización de sus fondos. Esto ha permitido, junto al desarrollo de buscadores -simples y avanzados- como herramientas virtuales, el conocimiento de numerosos datos recogidos en estas publicaciones, que albergan entre sus páginas una ingente cantidad de información.

Para el caso de España son esenciales las colecciones hemerográficas alojadas en la Biblioteca Virtual de Prensa Histórica y la Hemeroteca Nacional de España. Además contamos con las hemerotecas de los medios gráficos vigentes y de las 
empresas de comunicación, así como los de las colecciones de las administraciones locales y autonómicas que, por desgracia, no son en todos los casos de fácil acceso a través de internet, o bien no se encuentran digitalizadas y requieren en muchos casos una tediosa aunque necesaria consulta física.

\section{CONCLUSIONES}

El periodo comprendido entre la segunda mitad del siglo XIX y la Restauración constituye un periodo decisivo en la historia de la educación contemporánea española en el que la hemerografía histórica se presenta como una fuente significativa para su análisis y estudio, bien sea profesional o generalista.

Esta última presenta una gran cantidad de información a través de referencias directas e indirectas, además de infinidad de datos contextuales.

La prensa en general constituye un elemento característico y definitorio de la sociedad decimonónica y de la Restauración y, como tal, deben establecerse una serie de pautas de investigación adecuadas que nos permitan abordar los contenidos fundamentales relacionados con la historia de la educación.

Las especiales características de la hemerografía, y su conexión con el devenir socio-político y social hacen necesaria una reflexión acerca de las necesidades formativas del investigador que desea interpretar la información allí contenida.

La formación del investigador de la educación ha de ser diversa y abarcar diferentes áreas de conocimiento, sobre todo en aquellos casos en los que tratemos con prensa generalista. Esta fuente exige una especial atención a los elementos coyunturales y, por ello, demanda un acercamiento a la misma desde diversas disciplinas, atendiendo esecialmente a una amplia y variada preparación en el ámbito de las humanidades, así como en ciencias sociales.

La mejor abordabilidad de las fuentes hemerográficas en la actualidad propicia el uso de este recurso como fuente histórica. Su utilización no deja de incrementarse cada día gracias a las nuevas tecnologías, aumentando el corpus documental hemerográfico y su accesibilidad.

\section{REFERENCIAS BIBLIOGRÁFICAS}

Abadal, E. y Guallar, J. (2010). La prensa digital en las bibliotecas. Barcelona: Trea.

Almuñía Fernández, C. (1977). La prensa vallisoletana durante el siglo XIX. Valladolid: Diputación de Valladolid, Institución Cultural Simancas. 
Aróstegui Sánchez, J. (2004). La historia del presente: ¿una cuestión de método? En Navajas Zubeldía (Coord.), Actas del IV Simposio de Historia Actual (pp. 41-75). Logroño: Instituto de Estudios Riojanos.

Aróstegui Sánchez, J. (2001). La investigación histórica. Teoría y método. Barcelona: Crítica.

Arroyo Vázquez, M. a L. (2004). La prensa como fuente histórica. La percepción del modelo estadounidense. C. Navajas Zubeldía (Coord.), Actas del IV Simposio de Historia Actual (vol. 1, pp. 435-446). Logroño: Universidad Autónoma de Madrid.

Brioso y Mayral, J. (2001). Noticias Carlistas. El Diario de Huesca, 125 años después. Huesca: Instituto de Estudios Altoaragoneses, pp.55-84.

Bussy Genovois, D. (2002). La prensa. en La época de la Restauración (1875-1902), Civilización y cultura, Historia de España, Menéndez Pidal (vol. XXXVI, pp. 383384). Madrid: Espasa-Calpe.

Calero Delso, J. (2013). La educación en España en el siglo XIX. En Actas de las XI Jornadas de Castilla-La Mancha sobre Investigación en Archivos. La educación en España. Historia y Archivos., $19-22$ noviembre 2013 (pp. 315-328). Guadalajara, AHPGU. https://doi.org/10.35197/rx.09.03.e.2013.01.sh.

Cándido Ruiz, R. y Palacios Lis, I. (1983). Iglesia y educación en la España decimonónica: política concordataria (1851). Historia de la educación: Revista interuniversitaria, 2, pp. 287-298.

Checa Godoy, A. (2002). Historia de la prensa pedagógica. Universidad de Sevilla.

Checa Godoy, A. (2011). Historia de la prensa andaluza. Sevilla: Alfar.

Desvois, J. M. (1986). Historia de la prensa: el recurso del método. En La crisis de la Restauración. España, entre la primera guerra mundial y la II República, II Coloquio de Segovia sobre Historia Contemporánea dirigido por Manuel Tuñón de Lara. Madrid: Siglo XXI.

Duverger, M. (1996). Métodos de las Ciencias Sociales. Barcelona: Ariel.

Espejo Cala, C. (2013). La aparición del periodismo en Europa: Comunicación y propaganda en el Barroco. Madrid: Marcial Pons Historia. 
Ezpeleta Aguilar, F. (2015-2016). La prensa pedagógica del XIX como fuente para historiar la didáctica de la lengua. Ianua. Revista Philologica Romanica, 15-16, pp. 159-171.

González Sánchez, J. M. y Muñoz Romano, J. L. (2013). Fuentes documentales para el estudio de la educación en la Edad Contemporánea. En Actas de las XI Jornadas de Castilla-La Mancha sobre Investigación en Archivos. La educación en España. Historia y Archivos (pp. 258-312). Guadalajara, 19-22 noviembre.

Hernández Díaz, J. M.a (2013). Prensa pedagógica y patrimonio histórico educativo en España. conceptualización y géneros textuales. En Prensa pedagógica y patrimonio histórico educativo: contribuciones desde la Europa mediterránea e Iberoamérica (pp. 12-32). Salamanca: Ediciones Universidad de Salamanca.

Hernández Díaz, J. M.a (2015). La prensa de los escolares y estudiantes. Su contribución al patrimonio histórico educativo. Salamanca: Ediciones Universidad de Salamanca.

Hernández Díaz, J. M. a (1984). Iniciación a la Historia de la educación en Castilla León. Salamanca: Instituto de Ciencias de la educación, Universidad de Salamanca.

Hernández Ramos, P. (2017). Consideración teórica sobre la prensa como fuente historiográfica. Historia y comunicación social, 22(2), pp. 463-477. https://doi. org/10.5209/HICS.57855.

Hibbs-Lissorgués, S. (1995). Iglesia, prensa y sociedad en España (1868-1904), Alicante: Instituto Alicantino de Cultura Juan Gil-Albert.

Jover Zamora, J. M,a (1993). La época de la Restauración: Panorama político-social, 1875- 1902. En VV. AA., Revolución burguesa, oligarquía y constitucionalismo, vol. VIII de la Historia de España (oo, 271-273) Barcelona: Labor.

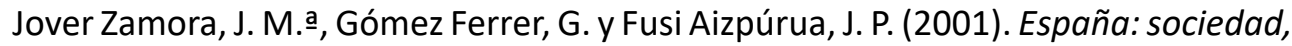
política y civilización, siglos XIX y XX. Barcelona: Areté.

Juan Borroy, V. M. (2004). La tarea de Penélope. Cien años de escuela pública en Aragón. Zaragoza: Biblioteca Aragonesa de Cultura, Institución Fernando El Católico.

Lozano, J. (2015). El discurso histórico. Madrid: Sequitur.

Marcuello Benedicto, I. (1999). La libertad de imprenta y su marco legal en la España liberal. Ayer, revista de historia contemporánea, 34, pp. 65-92. 
Medina Medina, A. (2012). La formación del sistema educativo español. En O. Negrín Fajardo (Coord.), Historia de la Educación Española. Madrid: UNED.

Mediavilla Gutiérrez, J. M. (2006). Los orígenes del periodismo educativo en Toledo (1866-1868). El prisma moderado. Ayuntamiento de Toledo.

Miguel Alonso, A. (1990). Del Plan Pidal a la la Ley Moyano: consolidación de la biblioteca de la Universidad Central. En Estudios históricos: Homenaje a los profesores José M. a Jover Zamora y Vicente Palacio Atard (vol. 2, pp. 681-702). Madrid: Universidad Complutense.

Moradiellos García, E. (2011). El oficio de historiador. Madrid: Siglo XXI.

Moradiellos García, E. (2016). Historia mínima de la Guerra Civil española. Madrid: Turner.

Montero Alcaide, A. (2009). La ley de Instrucción Pública (Ley Moyano, 1857). Cabás: Revista del Centro de Recursos, Interpretación y Estudios en materia educativa (CRIEME) de la Consejería de Educación del Gobierno de Cantabria (España) [publicación seriada en línea], 1. http://revista.muesca.es/articulos/71-la-leyde-instruccion-publica-ley-moyano-1857.

MORENO FERNÁNDEZ, C. M.ạ (2010). La herencia educativa de Andrés Manjón: aprender jugando en las escuelas del Ave María. Cabás: Revista del Centro de Recursos, Interpretación y Estudios en materia educativa (CRIEME), 4. http:// revista.muesca.es/articulos4/167-la-herencia-educativa-de-andres-manjonaprender-jugando-en-las-escuelas-del-ave-maria?showall=1.

Nasarre López, J. M.a (2001). La enseñanza en El Diario de Huesca. El Diario de Huesca, 125 años después. Huesca: Instituto de Estudios altoaragoneses.

Navarro Pérez, J. (2002). La contribución de J.G. Droysen a la filosofía de la historia y a la hermenéutica. Estudios filosóficos, 51(146), pp. 39-67.

Ramón Salinas, J. (2014) Ocio y cultura en Huesca durante la Restauración (18751902) a través de las publicaciones periódicas locales. Zaragoza: Zaguán, Universidad de Zaragoza.

Ruíz Berrio, J. (1980). Los Congresos Pedagógicos en la Restauración. Bordón. Revista de pedagogía, 234, pp. 401-422. 
Ruíz Rodrigo, C. y Palacios Lis, I. (1983). Iglesia y educación en la España decimonónica: política concordataria (1851). Historia de la educación: Revista interuniversitaria, 2, pp. 287-298.

Sáiz García, M. a D. (1996). Nuevas fuentes historiográficas. Historia y comunicación social, 1, pp. 131-144.

Salinas Zárate, M.a E. (1995). Aproximación a la historia urbana de Logroño a través de la prensa periódica local. Artigrama, revista del departamento de Historia del Arte, 11, pp. 280-281.

Sarasúa, C., (2002). Aprendiendo a ser mujeres: las escuelas de niñas en la España del siglo XIX. Cuadernos de Historia Contemporánea, 24, pp. 281-297.

Seoane Couceiro, M.a C. (1996). Historia del periodismo en España, vol.2. El siglo $X I X$. Madrid: Alianza Universidad Textos.

Teruel Melero, M.a P. (2001). Referencias pedagógicas desde El Diario de Huesca (1875-1882). En El Diario de Huesca, 125 años después. Huesca: Instituto de Estudios altoaragoneses.

Tiana Ferrer, A. (1994). La escuela privada. En J.-L. Guereña et al. (Eds.), Historia de la Educación en la España Contemporánea. Diez años de investigación. CIDE, Ministerio de Educación y Ciencia.

Tuñón de Lara, M. (1974). Metodología de la historia de la prensa española. Madrid: Siglo XXI.

Van Dijk, T. A. (2011). Sociedad y discurso: cómo influyen los contextos sociales sobre el texto y la conversación. Barcelona: Gedisa.

Van Dijk, T. A. (2017). Discurso y contexto. Barcelona: Gedisa.

Van Dijk, T. A. (2003). Ideología y discurso. Barcelona: Ariel.

Viñao Frago, A. (1996). Lenguaje y realidad. El discurso histórico y su aplicación al ámbito histórico-educativo. Anales de Pedagogía, 14, pp. 157-214.

Vivanco Saavedra, L. I. (2000). Características esenciales del pensamiento historiográfico de Ibn Jaldún. Opción: Revista de Ciencias Humanas y Sociales, 31, pp. 27-43. 